\title{
Polyunsaturated Fatty Acids as Dietary Supplements: Biological Activities and Sources
}

\author{
Derell Hardman and Stylianos Fakas
}

Department of Food and Animal Sciences, Alabama A\&M University, Normal, AL 35762, USA

\begin{abstract}
Polyunsaturated fatty acids (PUFAs) have been used as dietary supplements for quite some time with various health claims. Major dietary PUFAs are classified as omega- 3 and omega- 6 based on their structure, and these fatty acids are considered essential nutrients. The two classes of PUFAs, although structurally similar, present different biological activities that can be beneficial or detrimental to humans. Some of these biological activities have been known for a long time, while others are emerging from the plethora of dietary studies done on PUFAs. Yet many of the health claims for PUFAs are still debated, with the findings being conflicting. This review summarizes our current knowledge on the biological activities of PUFAs and discusses the dietary sources available. In light of new dietary recommendations, PUFA consumption is on the rise, so new dietary sources for these fatty acids are needed.
\end{abstract}

\author{
Publication History: \\ Received: July 13, 2016 \\ Accepted: November 26, 2016 \\ Published: November 28, 20166
}

\section{Keywords:}

Polyunsaturated fatty acids, Dietary supplements, omega-6, omega-3, Microbial oils, HDL subfraction,

\section{Introduction}

Polyunsaturated fatty acids (PUFAs) are long chain fatty acids (i.e. carbon chain length is more than 18 carbon atoms) that constitute a group of biologically active compounds essential to humans. The position of the double bond that is closest to the methyl end of the fatty acid chain classifies PUFAs into two groups: the omega-3, where this double bond is found three carbon atoms away from the methyl group, and the omega- 6 , where the double bond is six carbon atoms away from the methyl group. These two groups, although structurally similar, have very different biological activities [1-3]. Ongoing research has shown that omega-3 PUFAs have a beneficial effect, while omega- 6 could contribute to diseases like obesity, inflammation and cardiovascular disease [3-9]. Even though information is widely available on the benefits of PUFAs in the diet, most people do not consume PUFAs on a regular basis. This may be due to the less availability of PUFA sources due to location or socio-economic reasons [10]. However, the market for PUFAs is expected to expand by 2020 as the health-consciousness among the consumers increases [11]. Fish, particularly oily fish such as salmon and mackerel, and fish oils are the major dietary sources of PUFAs in most countries. However, fish are not a sustainable source because of decreasing amounts and the contamination of their habitats which affects the quality of the final product (i.e. fish or fish oil) $[12,13]$. Therefore, alternative sources are needed to meet the recommended daily amounts.

Microbial lipids have long been considered as sources of PUFAs [14-16]. Indeed, microbial lipids can be produced from simple sugars like glucose under well controlled conditions in a sustainable manner $[17,18]$. The major drawback of microbial lipids is their high production costs which are limiting their market penetration. Genetic engineering of microorganisms that would increase their lipid productivity could alleviate the costs and provide a sustainable and affordable source of PUFAs [19, 20]. Overall, the growing PUFA market is expected to increase the demand for these products so novel lipids, whether of microbial or other origin, may soon hit the market.

In this review, the major biological activities of omega-3 and omega-6 PUFAs will be discussed and an overview of their dietary sources will be given.

\section{Polyunsaturated Fatty Acid Biosynthesis}

The biosynthesis of PUFAs involves a series of elongation and desaturation reactions that convert one of the final products of the the fatty acid biosynthetic pathway, stearic acid, to the different omega- 6 and omega- 3 fatty acids (Figure 1). Stearic acid is desaturated to produce the monounsaturated fatty acid, oleic acid (C18:1), which can be considered as the biosynthetic precursor for both series of PUFAs. Oleic acid is further desaturated to yield linoleic acid (C18:2), the first member of the omega- 6 series. Linoleic acid sits at a branching point of the pathway and can be desaturated either to $\gamma$-linolenic acid (18:3-omega-6) or $a$-linolenic acid (18:3-omega-3) [8, 21, 22]. The latter reaction is catalyzed by $\Delta 15$-desaturase, also known as omega- 3 desaturase, because it introduces a double bond between the omega-2 and omega-3 carbon atoms. a-linolenic acid is the biosynthetic precursor of the omega-3 PUFAs giving rise to the long chain eicosapentaenoic acid (EPA, 20:5-omega-3) and docosahexaenoic acid (DHA, 22:6-omega-3). On the other hand, $\gamma$-linolenic acid is further elongated and desaturated to yield arachidonic acid (AA, 20:4), the major omega-6 PUFA.

\section{Dietary significance of PUFAs}

\section{Cardiovascular disease}

According to AHA, cardiovascular disease (CVD) remains the leading cause of death with17.3 million deaths per year globally [5]. The predictions are overwhelming: more than 23.6 million people are expected to die from CVD by the year 2030. CVD is described as diseases of the blood vessels and circulatory system, including coronary heart disease, myocardial infarction, and stroke. Dietary interventions for decreasing CVD risks have long been sought and are the subject of many epidemiological and clinical studies. Among these interventions, supplementation of diets with omega-3 PUFAs has attracted a lot of interest, however, the results remain contradictory. Both DHA and EPA along or in combination have been used

"Corresponding Author: Prof. Stylianos Fakas, Department of Food and Animal Sciences, Alabama A\&M University, Normal, AL 35762, USA; E-mail: stylianos.fakas@aamu.edu

Citation: Hardman D, Fakas S (2016) Polyunsaturated Fatty Acids as Dietary Supplements: Biological Activities and Sources. Int J Clin Nutr Diet 2: 113. doi: https://doi.org/10.15344/ijcnd/2016/113

Copyright: (c) 2016 Hardman et al. This is an open-access article distributed under the terms of the Creative Commons Attribution License, which permits unrestricted use, distribution, and reproduction in any medium, provided the original author and source are credited. 
as a supply of omega-3 PUFAs in dietary intervention studies. Epidemiological studies in China showed that increased intake of dietary omega-3 PUFAs from marine and plant sources is associated with reduced risk of CVD mortality [23]. These results are similar to the results of the JELIS study performed in Japan which looked at the long-term effects of EPA supplementation on the prevention of major coronary events in hypercholesterolemic patients [24]. The results of this study showed that supplementation with EPA reduced major coronary events which indicates that EPA could be used as a treatment for CVD [24]. On the other hand, several studies failed to show an effect of omega-3 supplementation on CVD [4, 25]. Similar results were obtained with systematic data analysis of 20 published epidemiological studies where no correlation between omega-3 supplementation and CVD was found [26]. These results, however, should be interpreted with caution since it has been shown that several factors may affect the outcome of studies like the dose of omega-3 PUFAs, the baseline status of omega- 3 fatty acids of the participants in the study, and the bioavailability of PUFAs which seems to be affected by the fat content of the meal [7].

\section{Inflammation}

Omega-3 fatty acids have anti-inflammatory effects, which are mediated by antagonizing the pro-inflammatory omega- 6 fatty acids. Omega- 6 fatty acids reside in the membranes of inflammatory cells (i.e. leucocytes, neutrophils) where they are esterified in the membrane phospholipids. The inflammatory reaction cascade starts with the action of phospholipase A2 on membrane phospholipids which releases omega- 6 fatty acids, such as arachidonic acid [8]. The arachidonic acid is then acted upon by oxidative enzymes like cyclo-oxygenase (COX) and lipoxygenase (LOX) which catalyze the first steps of the biosynthetic pathways that lead to the production of prostaglandins (i.e. PGE2) and thromboxanes (COX), and leucotrienes (LOX) [8]. These compounds belong to the group termed eicosanoids and are involved in inflammation.

Omega-3 PUFAs such as EPA and DHA can compete with arachidonic acid for the available COX and LOX enzymes because omega-3 PUFAs are also a substrate for these enzymes. The key difference is that the metabolites that are produced by the action of COX and LOX on EPA and DHA are much less potent in inducing inflammation than those produced from arachidonic acid. This means that less inflammatory molecules should be produced in the presence of EPA and DHA. Indeed, a recent study showed that dietary omega-3 PUFAs (i.e. EPA and DHA) can modulate inflammatory markers, but DHA was proven to be more potent than EPA [9]. The mechanism by which omega-3 PUFAs exert their anti-inflammatory effects seems to be through G protein-coupled receptors like GPR120 which mediates the inhibition of pro-inflammatory signaling pathways [27].

\section{Obesity}

Obesity is a growing epidemic in developing countries where Western diet is predominant [28]. Dietary interventions have long been considered as a means to prevent obesity and in these interventions, omega-3 fatty acids have shown promise. Studies with rodents fed with high fat diets that contain large amounts of omega-3 PUFAs show a consistent decrease in the obesity of the animals [28]. In humans, however, similar supplementation studies have produced conflicting results [29]. These discrepancies are thought to be due to inconsistency in the design of the studies, the form of supplementation (i.e. fish oil versus omega-3 supplements), genetic variations among

Int J Clin Nutr Diet the participants, variations in weight and state of disease (lean versus obese individuals) etc. [3]. Furthermore, the mechanisms by which omega-3 exert their anti-obesity effects are not well understood. For example, a study on the anti-obesity effects of omega-3 fatty acids showed that they can act by increasing satiety, thus restricting food intake in overweight and obese individuals [30]. Other studies found that omega- 3 fatty acids act by inhibiting the lipogenic enzymes, while at the same time they increase fatty acid oxidation [31]. Clearly, more studies are needed to support the anti-obesity effect of omega-3 PUFAs.

On the other hand, a positive correlation between omega- 6 intake and increased body fat mass has been established in recent years $[6,32,33]$. For example, the increasingly high intake of linoleic acid that is characteristic of Western diets is correlated with increased rates of obesity [34]. The mechanism by which linoleic acid is contributing to weight gain and obesity seems to be through its oxidized metabolites 9-hydroxy-octadecadienoic (9-HODE) and 13-hydroxy-octadecadienoic (13-HODE) which are thought to trigger preadipocyte (fat cell) differentiation [34]. Also, linoleic acid can be converted to arachidonic acid in the body which is correlated with increased inflammation (see above) and increased adipocyte formation [34]. In mice, a diet high in linoleic acid increased body fat mass and this increase was seen in successive generations that were fed the same diet [1]. Thus, it seems that increased exposure to omega- 6 fatty acids can increase adiposity across generations, which is in line with the increasing obesity epidemic.

\section{Prenatal and infant nutrition}

Perhaps one of the most widely recognized health benefits of omega-3 PUFAs is in prenatal and infant nutrition. Human brains can accumulate DHA up to the age of eighteen, but most of the DHA accumulates during pregnancy until the age of two [35]. The Western diet does not provide sufficient amounts of omega-3 PUFAs to the mother and thus the growing fetus, so some form of dietary supplementation is needed during pregnancy [36]. Research findings suggest that dietary supplementation with EPA and DHA during pregnancy reduces the risk of preterm birth, increases the birth size [37] and improves the cognitive and visual development of the infant [38]. However, a recent systematic data analysis failed to show any positive correlation between infant brain development and omega-3 supplementation of the maternal diet [39]. Furthermore, supplementation with DHA during pregnancy does not improve the attention or working memory of preschoolers [36]. In infant nutrition, early studies showed that supplementation of infant formulas with EPA and DHA improved cognitive and visual development of the infants [40-42]. Thus, dietary recommendations for pregnant women and infants include some form of omega- 3 supplementation.

\section{Cognitive performance and mood disorders in adults}

Despite the conflicting evidence on the actual effect of omega-3 supplementation on brain development in infants, there is ongoing interest in researching the effect of this supplementation all the way from childhood to adulthood and to late stages in life. Systematic reviews and meta-analysis of published research show a correlation between DHA and EPA supplementation and memory function in older adults [43]. In populations that are PUFA deficient, supplementation with omega-3 shows some benefit in cognitive functions [44]. As cognitive aging progresses, the onset of certain memory related diseases, such as dementia and Alzheimer's, begins. Since omega-3 PUFAs cause an 
improvement of cognitive performance, it has been argued that they could also contribute to the prevention of these diseases. Indeed, supplementation of elderly diets with $2.2 \mathrm{~g}$ per day of omega-3 PUFAs significantly improved memory performance [45]. Recent studies have focused on the effect of PUFA supplementation on mood disorders and other psychiatric diseases. There is limited evidence that suggest a beneficial effect of omega-3 PUFAs on depression where they decrease the symptoms [10]. Furthermore, studies have showed that individuals at risk of developing psychotic disorders might benefit from omega-3 supplementation [2]. Overall, there seems to be a positive effect of omega-3 PUFA on cognitive performance and mood enhancement across ages.

The role of PUFAs in certain diseases is becoming more apparent because of the increasing number of well-controlled studies that are addressing the effects of dietary PUFAs. There is increasing evidence that suggest that a deficit in omega-3 PUFAs is linked to diseases such as CVD, while an imbalance in omega- 3 to omega- 6 ratio can lead to inflammation and obesity. Also, cognitive performance throughout life seems to benefit by supplementation of diets with omega-3 PUFAs. These findings have been translated to dietary recommendations that are discussed below.

\section{Dietary Sources of PUFAs}

\section{Conventional dietary sources}

In an effort to reduce the risk of CVD in the United States, the American Heart Association (AHA) recommends the consumption of fish, particularly oily fish, at least twice a week in the diets of adults and children over two years old [46]. The dietary recommendations for omega- 3 fatty acids in the form of EPA and DHA are 0.5 to $1 \mathrm{~g}$ per day for individuals with normal blood triglyceride levels. In pathological conditions such as hypertriglyceridemia, AHA recommends that the daily dose should be increased to 2-4 g of EPA and DHA [47]. Dietary sources of omega-3 PUFAs include plants (e.g. leafy vegetables) and oily fish (e.g. salmon, mackerel). These sources are particularly enriched in some dietary regimens, like the Mediterranean diet.

Mediterranean diet has long been considered as a good source of PUFAs. Adherence to Mediterranean diet has been linked to health benefits such as a lower likelihood of incident cognitive impairment [48], and less brain atrophy in older adults [49]. Mediterranean diet has also been shown to protect against CVD [50], and reduce the mortality rates in patients that suffered a myocardial infarction [51]. These health benefits were attributed, at least partly, to the omega-3 PUFAs that are found in fish and vegetables [52]. Indeed, intervention studies showed that switching to a Mediterranean diet increases the levels of omega-3 PUFAs in plasma fatty acids [53] and in serum [54]. The major source of long chain omega-3 PUFAS in Mediterranean diets is fish which are consumed at moderate to high amounts in different areas of the Mediterranean basin [55, 56]. Fish consumption, however, is associated with potential health problems and sustainability issues.

The problems related to fish being the primary source of PUFAs include less sustainability because of the decline of wild fish populations and the potential for heavy metal contamination. Some compounds (e.g. methyl mercury and polychlorinated biphenyls) can contaminate fish and become a concern for specific groups of the population, such as children and pregnant women [46]. Furthermore, the AHA recommends that consumers check with the Federal Drug Administration(FDA)and theEnvironmentalProtection Agency(EPA) before consuming fish from local sources to ensure that it isn't potentially contaminated with any heavy metals [46]. Because of the problems (i.e. sustainability and potential heavy metal contamination) associated with fish as a source of PUFAs, more sustainable and safer sources of these oils needs to be found.

\section{Microbial oils as sources of PUFAs}

Microbial oils have long been considered as an alternative to fish oils because they contain high amounts of PUFAs $[16,57]$. These are the oils that are produced from fungi $[58,59]$ and algae $[59,60]$ and their composition is pretty similar to vegetable and fish oils. The final product (i.e. purified microbial oil) is made almost entirely of triacylglycerol and can be used as a dietary supplement [19]. The advantage of microbial oils is that they can be produced under well controlled conditions in a bioreactor and thus the final product can meet the highest standards of purity and safety required for human nutrition [61]. The process for microbial oil production is usually heterotrophic, meaning that a carbon source like glucose is supplied to the microorganism. The glucose is consumed and converted to biomass until an essential nutrient, usually nitrogen, runs out. Since nitrogen is essential for growth, biomass production ceases after nitrogen is exhausted, and the microbial cells enter an oil accumulating phase [61]. When oil accumulation reaches its maximum value, the microbial cells are harvested and the oil is extracted using the same procedures used for vegetable oils [62]. However, microbial oils are much more expensive to produce than vegetable oils and thus only specialty oils with high nutritional value can be economically produced.

Commercial success for microbial oils came in the early 90s where large-scale processes for the production of a DHA rich oil by algae were developed by Martek Biosciences in USA. This oil was used in infant formulas and quickly became a success mainly due to the lack of alternative sources [63]. The oil produced is called DHASCO ${ }^{\text {ma }}$ or life's DHA and it received GRAS status by FDA in 2002. The annual sales of this oil were between 2,000 to 3,000 tons in 2011 and are probably close to 6,000 tons today [17]. The market for novel microbial oils remains open because of the new research findings that support the various benefits that come from PUFA supplementation. A good example of this is EPA rich oils, which are sought by those seeking to improve their mood and overall feeling of well-being. Indeed, an EPA rich oil has been developed using a genetically modified yeast, Yarrowia lipolytica [19]. Yeasts do not produce PUFAs like EPA naturally and thus they need to be engineered to be able to produce them. The yeast EPA oil was sold under the name New Harvest and was marketed as a vegetarian alternative to fish oils presenting claims of improved mood and overall well-being. However, this oil is currently off market. An alternative microbial source would be microalgae which are known to synthesize long chain PUFAs. Indeed, DHA from microalgae has been used for supplementing infant formulas for the past 25 years $[14,16$, 63]. Also, processes for commercial EPA production by microalgae have been sought, but the economics do not look favorable $[59,60]$. However, microbial lipids are well positioned to fulfill the growing needs of the PUFA market.

\section{Conclusion}

PUFAs are expected to play major role in dietary interventions designed to protect against a variety of diseases (CVD, obesity etc.) and improve cognitive performance. As more people are becoming aware of the PUFA health benefits, the demand for these fatty acids is 
Citation: Hardman D, Fakas S (2016) Polyunsaturated Fatty Acids as Dietary Supplements: Biological Activities and Sources. Int J Clin Nutr Diet 2: 113. doi: https://doi.org/10.15344/ijcnd/2016/113

Page 4 of 5

expected to increase in the next years. New sources of PUFAs will then be needed and microbial lipids are well positioned to fill the need.

\section{Acknowledgments}

This work was supported by the Evans-Allen Grant no.1005735 and Capacity Building Grants Program Grant no. 1007503 from the USDA National Institute of Food and Agriculture.

\section{References}

1. Massiera F, Barbry P, Guesnet P, Joly A, Luquet S, et al. (2010) A Westernlike fat diet is sufficient to induce a gradual enhancement in fat mass over generations. J Lipid Res 51: 2352-2361.

2. Saah T, Garlow SJ, Rapaport MH (2015) Polyunsaturated fatty acids in adult psychiatric disorders: A comprehensive overview. In: Immunology and Psychiatry: From Basic Research to Therapeutic Interventions. (Eds) Müller N, Myint A-M, Schwarz JM. Cham: Springer International Publishing, pp. 371-395

3. Simopoulos AP (2016) An Increase in the Omega-6/Omega-3 Fatty Acid Ratio Increases the Risk for Obesity. Nutrients 8: 128.

4. Kromhout D, Giltay EJ, Geleijnse JM; Alpha Omega Trial Group (2010) n-3 fatty acids and cardiovascular events after myocardial infarction. $\mathrm{N}$ Engl J Med 363: 2015-2026.

5. Mozaffarian D, Benjamin EJ, Go AS, Arnett DK, Blaha MJ, et al. (2016) Executive Summary: Heart Disease and Stroke Statistics-2016 Update: A Report From the American Heart Association. Circulation 133: 447-454.

6. Muhlhausler BS, Ailhaud GP (2013) Omega-6 polyunsaturated fatty acids and the early origins of obesity. Curr Opin Endocrinol Diabetes Obes 20: $56-61$.

7. von Schacky C (2015) Omega-3 fatty Acids in cardiovascular disease-An uphill battle. Prostaglandins, Leukotrienes Essent. Prostaglandins Leukot Essent Fatty Acids 92: 41-47.

8. Calder PC (2013) Omega-3 polyunsaturated fatty acids and inflammatory processes: nutrition or pharmacology? Br J Clin Pharmacol 75:645-662.

9. Allaire J, Couture P, Leclerc M, Charest A, Marin J, et al. (2016) A randomized, crossover, head-to-head comparison of eicosapentaenoic acid and docosahexaenoic acid supplementation to reduce inflammation markers in men and women: the Comparing EPA to DHA (ComparED) Study. Am J Clin Nutr 104: 280-287.

10. Giles GE, Mahoney CR, Kanarek RB (2013) Omega-3 fatty acids influence mood in healthy and depressed individuals. Nutr Rev 71: 727-741.

11. Markets and Markets (2016) Omega-3 PUFA Market by Type (DHA, EPA ALA), Application (Dietary Supplements, Functional Foods \& Beverages, Pharmaceuticals, Infant Formula), Source (Marine, Plant), Sub-source), \& Region - Global Forecasts to 2020.

12. Hong MY, Lumibao J, Mistry P, Saleh R, Hoh E, et al. (2015) Fish oil contaminated with persistent organic pollutants reduces antioxidant capacity and induces oxidative stress without affecting its capacity to lower lipid concentrations and systemic inflammation in rats. J Nutr 145: 939-944.

13. Gribble MO, Karimi R, Feingold BJ, Nyland JF, O'Hara TM, et al. (2016) Mercury, selenium and fish oils in marine food webs and implications for human health. J Mar Biol Assoc U 96: 43-59.

14. Kyle DJ (2001)The Large-Scale Production and Use of a Single-Cell Oi Highly Enriched in Docosahexaenoic Acid. ACS Symposium Series 788: 92-117.

15. Kyle DJ (1997) Production and use of a single cell oil which is highly enriched in arachidonic acid. Lipid Technol 9: 116-121.

16. Kyle D, Sicotte V, Singer J, Reeb S (1992) Bioproduction of docosahexaenoic acid (DHA) by microalgae. In: Industrial Applications of Single Cell Oils. American Oil Chemists' Society, Champaign, Illinois. (Eds) Kyle D, Ratledge C. AOCS Publishing, pp. 287-300.

17. Ratledge C (2013) Microbial oils: an introductory overview of current status and future prospects. Oilseeds and fats, Crops and Lipids 20: D602.
18. Fakas S, Papanikolaou S, Galiotou-Panayotou M (2009) Biochemistry and biotechnology of single cell oil. In: New Horizons in Biotechnology. (Eds) Pandey A, Larroche C. Delhi: Asiatech Publishers, pp. 38-60.

19. Xie D, Jackson EN, Zhu Q (2015) Sustainable source of omega-3 eicosapentaenoic acid from metabolically engineered Yarrowia lipolytica: from fundamental research to commercial production. Appl Microbiol Biotechnol 99:599-1610.

20. Fakas S (2016) Lipid biosynthesis in yeasts: A comparison of the lipid biosynthetic pathways between the model non-oleaginous yeast Saccharomyces cerevisiae and the model oleaginous yeast Yarrowia lipolytica. Eng Life Sci.

21. Certik M, Shimizu S (1999) Biosynthesis and regulation of microbial polyunsaturated fatty acid production. J Biosci Bioeng 87: 1-14.

22. Ratledge C, Wynn JP (2002) The biochemistry and molecular biology of lipid accumulation in oleaginous microorganisms. Adv Appl Microbiol 51: $1-51$.

23. Koh AS, Pan A, Wang R, Odegaard AO, Pereira MA, et al. (2015) The association between dietary omega-3 fatty acids and cardiovascular death: the Singapore Chinese Health Study. Eur J Prev Cardiol 22: 364-372.

24. Yokoyama $M$, Origasa $H$, Matsuzaki $M$, Matsuzawa $Y$, Saito $Y$, et al. (2007) Effects of eicosapentaenoic acid on major coronary events in hypercholesterolaemic patients (JELIS): a randomised open-label, blinded endpoint analysis. Lancet 369: 1090-1098.

25. Rauch B, Schiele R, Schneider S, Diller F, Victor N, et al. (2010) OMEGA, a randomized, placebo-controlled trial to test the effect of highly purified omega-3 fatty acids on top of modern guideline-adjusted therapy after myocardial infarction. Circulation 122: 2152-2159.

26. Rizos EC, Ntzani EE, Bika E et al. (2012) Association between omega-3 fatty acid supplementation and risk of major cardiovascular disease events: A systematic review and meta-analysis. JAMA 308:1024-1033.

27. Oh DY, Talukdar S, Bae EJ, Imamura T, Morinaga H, et al. (2010) GPR120 Is an Omega-3 Fatty Acid Receptor Mediating Potent Anti-inflammatory and Insulin-Sensitizing Effects. Cell 142:687-698.

28. Newberry EP, Xie Y, Kennedy SM, Luo J, Davidson NO (2006) Protection against Western diet-induced obesity and hepatic steatosis in liver fatty acid-binding protein knockout mice. Hepatology 44: 1191-1205.

29. Abeywardena MY, Belobrajdic DP (2016) Long-chain omega-3 polyunsaturated fatty acids and obesity. In: Obesity: A Practical Guide. (Eds) Ahmad IS, Imam KS. Cham: Springer International Publishing, pp. 29-44.

30. Parra D, Ramel A, Bandarra N, Kiely M, Martínez JA, et al. (2008) A diet rich in long chain omega-3 fatty acids modulates satiety in overweight and obese volunteers during weight loss. Appetite 51: 676-680.

31. Ukropec J, Reseland JE, Gasperikova D, Demcakova E, Madsen L, et al. (2003) The hypotriglyceridemic effect of dietary n-3 FA is associated with increased ß-oxidation and reduced leptin expression. Lipids 38:1023-1029.

32. Wang L, Manson JE, Rautiainen S, Gaziano JM, et al. (2016) A prospective study of erythrocyte polyunsaturated fatty acid, weight gain, and risk of becoming overweight or obese in middle-aged and older women. Eur $\mathrm{J}$ Nutr 55: 687-697.

33. Wang $L$ (2016) Omega-3 and omega-6 fatty acids: Role in body fat gain and development of obesity. N A J Med Sci 8:163-171.

34. Naughton SS, Mathai ML, Hryciw DH, McAinch AJ (2016) Linoleic acid and the pathogenesis of obesity. Prostaglandins Other Lipid Mediat 125: 90-99.

35. Brenna JT, Carlson SE (2014) Docosahexaenoic acid and human brain development: evidence that a dietary supply is needed for optimal development. J Hum Evol 77: 99-106.

36. Gould JF, Makrides M, Colombo J, Smithers LG (2014) Randomized controlled trial of maternal omega-3 long-chain PUFA supplementation during pregnancy and early childhood development of attention, working memory, and inhibitory control. Am J Clin Nutr 99: 851-859.

37. Carlson SE, Colombo J, Gajewski BJ, Gustafson KM, Mundy D, et al. (2013) DHA supplementation and pregnancy outcomes. Am J Clin Nutr 97: 808-815.

38. Koletzko B, Boey CC, Campoy C, Carlson SE, Chang N, et al. (2014) Current Information and Asian Perspectives on Long-Chain Polyunsaturated Fatty Acids in Pregnancy, Lactation, and Infancy: Systematic Review and Practice Recommendations from an Early Nutrition Academy Workshop. Ann Nutr Metab 65: 49-80. 
Citation: Hardman D, Fakas S (2016) Polyunsaturated Fatty Acids as Dietary Supplements: Biological Activities and Sources. Int J Clin Nutr Diet 2: 113. doi: https://doi.org/10.15344/ijcnd/2016/113

Page 5 of 5

39. Gould JF, Smithers LG, Makrides M (2013) The effect of maternal omega-3 (n-3) LCPUFA supplementation during pregnancy on early childhood cognitive and visual development: a systematic review and meta-analysis of randomized controlled trials. Am J Clin Nutr 97:531-544.

40. Makrides M, Neumann M, Simmer K, Pater J (1995) Are long chain polyunsaturated fatty acids essential nutrients in infancy. Lancet 345: 1463 1468.

41. Gill I, Valivety R (1997) Polyunsaturated fatty acids, Part 1: Occurrence, biological activities and applications. Trends Biotechnol 15: 401-409.

42. Craig-Schmidt MC, Huang MC (1998) Interaction of infant formula with long-chain polyunsaturated fatty acids. In: Lipids in infant nutrition. Edited by: Huang Y-S, Sinclair AJ. Champaign, Illinois: AOCS Press, pp. 63-84.

43. Yurko-Mauro K, Alexander DD, Van Elswyk ME (2015) Docosahexaenoic acid and adult memory: a systematic review and meta-analysis. PLoS One 10: e0120391.

44. Cooper RE, Tye C, Kuntsi J, Vassos 2, Asherson P (2015) Omega-3 polyunsaturated fatty acid supplementation and cognition: A systematic review and meta-analysis. J Psychopharmacol 29: 753-763.

45. Külzow N, Witte AV, Kerti L, Grittner U, et al. (2016) Impact of Omega-3 Fatty Acid Supplementation on Memory Functions in Healthy Older Adults. J Alzheimers Dis 51: 713-725.

46. American HeartAssociation Nutrition Committee, Lichtenstein AH, Appel LJ, Brands M, Carnethon M, et al. (2006) Diet and lifestyle recommendations revision 2006: a scientific statement from the American Heart Association Nutrition Committee. Circulation 114: 82-96.

47. Miller M, Stone NJ, Ballantyne C, Bittner V, Criqui MH, et al. (2011) Triglycerides and cardiovascular disease a scientific statement from the American Heart Association. Circulation 123: 2292-2333.

48. Tsivgoulis G, Judd S, Letter AJ, Alexandrov AV, Howard G, et al. (2013) Adherence to a Mediterranean diet and risk of incident cognitive impairment. Neurology 80: 1684-1692.

49. Gu Y, Brickman AM, Stern Y, Habeck CG, Razlighi QR, et al. (2015) Mediterranean diet and brain structure in a multiethnic elderly cohort. Neurology 85: 1744-1751.

50. Estruch R, Ros E, Salas-Salvadó J, Covas MI, Corella D, et al. (2013) Primary prevention of cardiovascular disease with a Mediterranean diet. N Engl J Med 368: 1279-1290.

51. de Lorgeril M, Renaud S, Mamelle N, Salen P, Martin JL, et al. (1994) Mediterranean alpha-linolenic acid-rich diet in secondary prevention of coronary heart disease. Lancet 343: 1454-1459.

52. Scoditti E, Capurso C, Capurso A, Massaro M (2014) Vascular effects of the Mediterranean diet-part II: role of omega-3 fatty acids and olive oil polyphenols. Vascul Pharmacol 63: 127-134

53. Féart C, Torrès MJ, Samieri C, Jutand MA, Peuchant E, et al. (2011) Adherence to a Mediterranean diet and plasma fatty acids: data from the Bordeaux sample of the Three-City study. Br J Nutr 106: 149-158.

54. Ambring A, Johansson M, Axelsen M, Gan L, Strandvik B, et al. (2006) Mediterranean-inspired diet lowers the ratio of serum phospholipid $n-6$ to n-3 fatty acids, the number of leukocytes and platelets, and vascular endothelial growth factor in healthy subjects. Am J Clin Nutr 83:575-581.

55. Willett WC, Sacks F, Trichopoulou A, Drescher G, Ferro-Luzzi A, et al (1995) Mediterranean diet pyramid: a cultural model for healthy eating. Am J Clin Nutr 61: 1402S-1406S.

56. Gil A, Gil F (2015) Fish, a Mediterranean source of n-3 PUFA: benefits do not justify limiting consumption. Br J Nutr 113 Suppl 2: S58-67.

57. Yazawa K, Watanabe K, Ishikawa C (1992) Production of eicosapentanoic acid from marine bacteria. In: Industrial applications of single cell oils Edited by: Kyle DJ, Ratledge C. Champaign: American Oil Chemists' Society, pp. 29-51.

58. Donot F, Fontana A, Baccou JC (2014) Single cell oils (SCOs) from oleaginous yeasts and moulds: Production and genetics. Biomass Bioenergy 68: 135-150.

59. Bellou S, Triantaphyllidou IE, Aggeli D, Elazzazy AM, Baeshen MN et al. (2016) Microbial oils as food additives: recent approaches for improving microbial oil production and its polyunsaturated fatty acid content. Curr Opin Biotechnol 37: 24-35.
60. Bellou S, Baeshen MN, Elazzazy AM, Aggeli D, Sayegh F, et al. (2014) Microalgal lipids biochemistry and biotechnological perspectives. Biotechnol Adv 32: 1476-1493.

61. Ratledge C (2010) Single Cell Oils for the 21st Century. In: Single Cell Oils. Edited by: Cohen Z, Ratledge C. Champaign, Illinois: AOCS Press pp. 1-20.

62. Ratledge $\mathrm{C}$, Streekstra H, Cohen Z, Fichtali J () Down-stream processing, extraction and purification of single cell oils. In: Single Cell Oils. Edited by: Cohen Z, Ratledge C. Champaign, Illinois: AOCS 